\title{
Correction to: Fear of COVID-19 Scale for Hospital Staff in Regional Hospitals in Mexico: a Brief Report
}

\author{
Benjamín García-Reyna ${ }^{1}$ - Gilberto Daniel Castillo-García ${ }^{2}$. \\ Francisco José Barbosa-Camacho ${ }^{3}$ - Guillermo Alonso Cervantes-Cardona ${ }^{4}$. \\ Enrique Cervantes-Pérez ${ }^{5}$. Blanca Miriam Torres-Mendoza ${ }^{6}$. \\ Clotilde Fuentes-Orozco ${ }^{3} \cdot$ Kevin Josue Pintor-Belmontes ${ }^{3}$. \\ Bertha Georgina Guzmán-Ramírez ${ }^{3}$ - Aldo Bernal-Hernández ${ }^{3}$. \\ Alejandro González-Ojeda ${ }^{3} \cdot$ Gabino Cervantes-Guevara ${ }^{1,6,7}$
}

Published online: 27 January 2021

(C) Springer Science+Business Media, LLC, part of Springer Nature 2021

\section{Correction to: Int J Ment Health Addiction https://doi.org/10.1007/s11469-020-00413-x}

The name of coauthor Aldo Bernal-Hernández was incorrectly given as Aldo HernándezBernal in this article as originally published.

The original article has been corrected.

Publisher's Note Springer Nature remains neutral with regard to jurisdictional claims in published maps and institutional affiliations.

The online version of the original article can be found at https://doi.org/10.1007/s11469-020-00413-X

Gabino Cervantes-Guevara

gabino_guevara@hotmail.com

1 Centro Universitario del Norte, Universidad de Guadalajara, Colotlán, Jalisco, Mexico

2 Sindicato Nacional de Trabajadores del Instituto Mexicano del Seguro Social, Sección III, Guadalajara, Jalisco, Mexico

3 Unidad de Investigación Biomédica 02, Hospital de Especialidades del Centro Médico Nacional deOccidente, Instituto Mexicano del Seguro Social, Guadalajara, Jalisco, Mexico

4 Departamento de Disciplinas Filosófico, Metodológicas e Instrumentales, Centro Universitario de Cienciasde la Salud, Universidad de Guadalajara, Guadalajara, Jalisco, Mexico

5 Departamento de Nutrición Clínica, Instituto Nacional de Ciencias Médicas y Nutrición“Salvador Zubirán", Ciudad de Mexico, Mexico

6 Departamento de Bienestar y Desarrollo Sostenible, Centro Universitario del Norte, Universidad deGuadalajara, Km. 191, México 45D No. 23, 46200 Colotlán, Jalisco, Mexico

7 Hospital Civil de Guadalajara“Fray Antonio Alcalde", Universidad de Guadalajara, Guadalajara, Jalisco, Mexico 